\title{
Formulation and pharmacokinetic evaluation of a paclitaxel nanosuspension for intravenous delivery
}

This article was published in the following Dove Press journal:

International Journal of Nanomedicine

I2 July 20 I I

Number of times this article has been viewed

\author{
Yonglu Wang ${ }^{1,4}$ \\ Xueming $\mathrm{Li}^{1,2^{*}}$ \\ Liyao $\mathrm{Wang}^{3}$ \\ Yuanlong $\mathrm{Xu}^{\prime}$ \\ Xiaodan Cheng' \\ Ping $\mathrm{Wei}^{4}$ \\ 'College of Pharmacy, Nanjing \\ University of Technology, \\ Nanjing; ${ }^{2}$ State Key Laboratory \\ of Materials-Oriented Chemical \\ Engineering, Nanjing University of \\ Technology,Nanjing; ${ }^{3}$ College of Life \\ Science, Anhui Agricultural University, \\ Hefei; ${ }^{4}$ College of Biotechnology and \\ Pharmaceutical Engineering, Nanjing \\ University of Technology, Nanjing, \\ People's Republic of China \\ *These authors contributed equally to \\ this work.
}

Correspondence: Xueming Li, Ping Wei No 5, Xinmofan Road, Nanjing 210009 , People's Republic of China

$\mathrm{Tel}+862583172066$

Fax +8625 58139416

Email xuemingli@njut.edu.cn,

weiping@njut.edu.cn
Abstract: Paclitaxel is a diterpenoid isolated from Taxus brevifolia. It is effective for various cancers, especially ovarian and breast cancer. Due to its aqueous insolubility, it is administered dissolved in ethanol and Cremophor ${ }^{\circledR}$ EL (BASF, Ludwigshafen, Germany), which can cause serious allergic reactions. In order to eliminate Cremophor EL, paclitaxel was formulated as a nanosuspension by high-pressure homogenization. The nanosuspension was lyophilized to obtain the dry paclitaxel nanoparticles (average size, $214.4 \pm 15.03 \mathrm{~nm}$ ), which enhanced both the physical and chemical stability of paclitaxel nanoparticles. Paclitaxel dissolution was also enhanced by the nanosuspension. Differential scanning calorimetry showed that the crystallinity of paclitaxel was preserved during the high-pressure homogenization process. The pharmacokinetics and tissue distribution of paclitaxel were compared after intravenous administration of paclitaxel nanosuspension and paclitaxel injection. In rat plasma, paclitaxel nanosuspension exhibited a significantly $(P<0.01)$ reduced area under the concentration curve $(A U C)_{0-\infty}\left(20.343 \pm 9.119 \mu \mathrm{g} \cdot \mathrm{h} \cdot \mathrm{mL}^{-1}\right.$ vs $\left.5.196 \pm 1.426 \mu \mathrm{g} \cdot \mathrm{h} \cdot \mathrm{mL}^{-1}\right)$, greater clearance $\left(2.050 \pm 0.616 \mathrm{~L} \cdot \mathrm{kg}^{-1} \cdot \mathrm{h}^{-1}\right.$ vs $\left.0.556 \pm 0.190 \mathrm{~L} \cdot \mathrm{kg}^{-1} \cdot \mathrm{h}^{-1}\right)$, and shorter elimination half-life (5.646 \pm 2.941 vs $3.774 \pm 1.352$ hours) compared with the paclitaxel solution. In contrast, the paclitaxel nanosuspension resulted in a significantly greater $\mathrm{AUC}_{0-\infty}$ in liver, lung, and spleen (all $P<0.01$ ), but not in heart or kidney.

Keywords: high-pressure homogenization, tissue distribution, surfactant

\section{Introduction}

Paclitaxel, the first of the microtubule stabilizing agents, is a diterpenoid derived from the bark of the Pacific yew tree (Taxus brevifolia).${ }^{1-3}$ Clinical trials have shown that paclitaxel has antitumoral activity, particularly against ovarian carcinoma, breast cancer, and nonsmall cell lung cancer. ${ }^{4}$ Paclitaxel has been hailed by the National Cancer Institute as the most significant advance in chemotherapy of the period 1970-1990. ${ }^{2}$

In view of its aqueous insolubility, paclitaxel has to be dissolved in a mixture of $50 \%$ ethanol and $50 \%$ Cremophor ${ }^{\circledR}$ EL (BASF, Ludwigshafen, Germany), which is a polyoxyethylated castor oil. Cremophor has been proved to be associated with a number of side effects, including hypersensitivity and neurotoxicity. ${ }^{5-8}$ The use of prophylactic steroids and histamine receptor antagonists as anti-allergic pre-medication may decrease the incidence and severity of acute hypersensitivity reactions. However, milder reactions have still been found to occur in $5 \%-30 \%$ of patients. ${ }^{9}, 10$

The extensive clinical use of paclitaxel was somewhat delayed due to the lack of appropriate delivery vehicles. There is a need for the development of alternating formulation of paclitaxel with good aqueous solubility and at the same time eliminating 
adverse reaction. Numerous attempts have been made to improve the solubility and pharmacological properties of paclitaxel, such as cosolvents, emulsions, micelles, liposomes, microspheres nanoparticles, cyclodextrins, pastes, and implants. ${ }^{1}$

An alternative formulation is a nanosuspension, which is a colloidal dispersion of nanosized drug particles produced by an appropriate size-reduction method and stabilized by a suitable stabilizer. ${ }^{11-13}$ According to Noyes-Whitney and Ostwald-Freundlich principles, the particle size in the nanometer range can lead to an increased dissolution velocity and saturation solubility for a nanosuspension, which is usually also accompanied by an increase in bioavailability. ${ }^{11,14,15}$ Nanosuspensions are useful dosage forms for poorly soluble drugs. ${ }^{13,16,17}$ Nanosuspensions may also show passive targeting similar to colloidal drug carriers after intravenous administration..$^{18}$ Following modification to the surface with special stabilizers, some active targeting may also be achieved in vivo with nanosuspensions. ${ }^{13}$

Techniques such as high-pressure homogenization and wet milling are often used to produce nanosuspensions of small size suitable for pharmaceutical uses. ${ }^{17,19-21}$ The highpressure homogenization technique has been extensively described with regard to particle size-reduction efficiency. ${ }^{20}$ As it is very simple, time-saving, and an organic solvent-free process, high-pressure homogenization presents advantages over other milling techniques. However, with high-pressure homogenization, the drug particles should be sufficiently small to pass through the homogenization gaps. ${ }^{17}$

In this study, high-pressure homogenization was evaluated for the paclitaxel nanosuspension preparation for intravenous administration. The paclitaxel nanosuspension was characterized for its particle size distribution and morphology. We also selected the surfactants and optimized the parameters of the homogenization procedure to produce a stable nanosuspension with small and narrow-distributed sizes. The paclitaxel crystalline state was studied before and following the high-pressure homogenization process to assess whether the initial crystalline state was preserved, which was important for long-term stability. To assess the in vivo properties of paclitaxel nanosuspension, the pharmacokinetics and tissue distribution were also investigated.

\section{Materials and methods Materials}

Raw material of paclitaxel and paclitaxel injection (taxol) was obtained from Nanjing Aosaikang Medical Group Co
Ltd (Nanjing, China). Norethisterone was provided by the National Institute for the Control of Pharmaceutical and Biological Products (Beijing, China). Poloxamer 188 was provided by BASF (Ludwigshafen, Germany). Polyethylene glycol 400 (PEG-400) and sodium carboxymethyl cellulose (CMC-Na) were purchased from Shantou Xilong Chemical Plant (Guangdong, China). Tween ${ }^{\circledR} 80$ was purchased from Sinopharm Chemical Reagent Co Ltd (Shanghai, China). Lecithin PC-90T was purchased from Shanghai Taiwei Medical Group Co Ltd (Shanghai, China). Tyloxapol was purchased from Shanghai Jingchun Chemical Reagent Co Ltd (Shanghai, China). Carbomer was purchased from Haidian Huiyou Co Ltd (Beijing, China). High-performance liquid chromatography (HPLC)-grade acetonitrile and methanol were obtained from Wanqing Chemical Reagent Co Ltd (Nanjing, China). All other chemicals and reagents were of analytical grade.

\section{Preparation of paclitaxel nanosuspension}

The paclitaxel nanosuspension was prepared by Ultra-Turrax ${ }^{\circledR}$ homogenization followed by high-pressure homogenization. Poloxamer 188 and PEG-400 were dissolved in $50 \mathrm{~mL}$ of water to obtain the aqueous surfactant solution. Paclitaxel powder $(50 \mathrm{mg})$ was dissolved in $4 \mathrm{~mL}$ of mixed solution containing ethanol $(2 \mathrm{~mL})$ and acetidin $(2 \mathrm{~mL})$ to form the oil phase solution, which was poured into the aqueous surfactant solution, with continuous mixing. The initial suspension was then evaporated to remove organic solvents and was subjected to a pre-homogenization with an Ultra Turrax T25 homogenizer (IKA Instruments Ltd, Staufen, Germany) at $10,000 \mathrm{rpm}$ for 10 minutes at room temperature. This coarse suspension was then circulated respectively for six cycles at 300,500 , and 800 bar pressure through the NS1001L2K high-pressure homogenizer (Niro Soavi S.p.A. Co, Parma, Italy) as a form of pre-milling. Finally, the suspension was homogenized for 20 cycles at 1000 bar until an equilibrium size was reached. The samples were withdrawn after the homogenization size-reduction steps for size-distribution analysis. The nanosuspension was lyophilized immediately to obtain a dry powder. The samples of nanosuspension were dispensed into a glass flask and frozen at $-80^{\circ} \mathrm{C}$ and then transferred to a YO-230 freeze-dryer (Thermo Savant, Waltham, MA), and dried at $-30^{\circ} \mathrm{C}$ for 36 hours with a vacuum degree of $200 \mathrm{~Pa}$. The lyophilized product was re-dispersed with water for intravenous administration. Re-dispersion was by manual shaking for 1 minute and the re-dispersion volume being equivalent to the original volume of the nanosuspension. 


\section{Characterization of paclitaxel nanosuspension}

Particle size and polydispersity index (PI) measurement

The mean particle size and the PI of the paclitaxel nanosuspension under different homogenization conditions were determined by dynamic light scattering with a Zetasizer $3000 \mathrm{HS}$ system (Malvern Instruments, Malvern, Worcestershire, UK). Three observations were recorded for each sample. The diameters reported were calculated using volume distribution from Gaussian distribution which indicated the width of a particle distribution.

\section{Zeta potential (ZP) analysis}

The $\mathrm{ZP}$ is a measure of the electric charge at the surface of the particles indicating the physical stability of colloidal systems. The ZP values being higher than $|30 \mathrm{mV}|$ indicate electrostatic long-term stability of aqueous dispersions. ${ }^{22}$ In this study, the ZP values were assessed by determining the particle electrophoretic mobility using ZP equipment (Brookhaven Instruments, Holtsville, NY). The nanosuspension samples for ZP values analysis were added to the small sample dispersion unit. Three observations were recorded for each sample.

\section{Morphology}

Morphological evaluation of paclitaxel nanoparticles was conducted by transmission electron microscopy. The samples were placed over a copper grid coated with carbon film and air-dried, and then were stained with $0.1 \%$ phosphotungstic acid. Finally, the samples were air dried and then observed with an H-7650 transmission electron microscope (Hitachi Ltd, Tokyo, Japan).

\section{Crystalline state evaluation}

Crystalline state evaluation was conducted by differential scanning calorimetry (DSC) to indicate any transformation to amorphous state during the high-pressure homogenization process. A heating rate of $10^{\circ} \mathrm{C}$ per minute was employed over the range of $50^{\circ} \mathrm{C}-300^{\circ} \mathrm{C}$. Samples were analyzed in standard aluminum sample pans, with an empty pan used as a reference. Thermograms of paclitaxel nanosuspension samples were recorded using a NETZSCH DSC-204 differential scanning calorimeter (Netzsch, Selb, Germany).

\section{Dissolution study}

A ZRS-8G dissolution apparatus (Tianjin Tianda Tianfa Technology Co Ltd, Tianjin, China) operating at a rotation speed of $75 \mathrm{rpm}$ was used for in vitro testing of paclitaxel dissolution. All dissolution tests were run in triplicate on an equivalent of $10 \mathrm{mg}$ of paclitaxel in nanosuspension. Phosphate buffered saline (PBS) (pH 7.4) with 1\% Tween 80 was used as the dissolution media. The volume and temperature of the dissolution medium were $250 \mathrm{~mL}$ and $37^{\circ} \mathrm{C} \pm 0.5^{\circ} \mathrm{C}$, respectively. At each predetermined sampling time, $1 \mathrm{~mL}$ of sample was withdrawn using sampling port attached with $0.22 \mu \mathrm{m}$ filter disc, and $1 \mathrm{~mL}$ blank dissolution medium was added back into the vessels through the sampling port. Samples were centrifuged, and the resulting supernatant was diluted in a mobile phase, and $20 \mu \mathrm{L}$ volume was injected into the HPLC for analysis.

\section{Stability study}

Storage stability was studied by storing the lyophilized nanosuspension samples at $4^{\circ} \mathrm{C}$ and room temperature for 3 months. Periodically, samples were removed, and the particle size, PI, and ZP were measured. In addition, paclitaxel stability in the nanosuspension was examined by determining the amount of parent drug remaining after specific storage periods.

\section{Pharmacokinetics study}

Male Sprague Dawley (SD) rats (six rats each group) weighing 230-270 g were obtained from the Laboratory Animal Center of Nanjing Medical University. The rats were acclimatized for at least 1 week before experimentation, fed with standard diet, and allowed water ad libitum. All animal experiments were evaluated and approved by the Animal Ethics Committee of Nanjing University of Technology. Rats received intravenous injection of paclitaxel $(10 \mathrm{mg} / \mathrm{kg})$ or paclitaxel nanosuspension $(10 \mathrm{mg} / \mathrm{kg})$ via the tail vein. At predetermined time points $(5,10,15$, and 30 minutes, and then $1,2,4,6,8$, and 12 hours), the rats were anesthetized with diethyl ether, and the blood was collected from the retro-orbital sinus into heparin $(10 \mu \mathrm{L}, 500 \mathrm{IU} / \mathrm{mL})$-treated tubes. The blood samples were immediately centrifuged at $3000 \mathrm{rpm}$ for 15 minutes to isolate the plasma. The collected plasma was stored at $-80^{\circ} \mathrm{C}$ in an ultra-low temperature freezer (Thermo Fisher Scientific, Waltham, MA) for further analysis.

To determine the paclitaxel concentration, $50 \mu \mathrm{L}$ anorethisterone $\left(25 \mu \mathrm{g} \cdot \mathrm{mL}^{-1}\right)$ was added to $100 \mu \mathrm{L}$ plasma followed by adding $1 \mathrm{~mL}$ diethyl ether. Each sample was vortexed for 5 minutes with a XW-80A vortex mixer (Qingpu Huxi Instrument Factory, Shanghai, China). After centrifugation (Centrifuge 5810R, Eppendorf, Hauppauge, NY) at 12,000 rpm for 10 minutes to precipitate the proteins, the supernatant was 
removed to clean test tubes and evaporated until dry under a stream of nitrogen (Hengao Technology Development Co, Tianjin, China) at $37^{\circ} \mathrm{C}$. Residues were dissolved in $100 \mu \mathrm{L}$ of mobile phase, and $20 \mu \mathrm{L}$ aliquots were injected into the HPLC for analysis.

\section{Tissue distribution study}

Male ICR (imprinting control region) mice (32 mice each group) weighing 18-27 g were obtained from the Laboratory Animal Center of Nanjing Medical University. The mice were acclimatized for at least 1 week before experimentation, fed with standard diet, and allowed water ad libitum. All animal experiments were evaluated and approved by the Animal Ethics Committee of Nanjing University of Technology. Mice in each group received an intravenous paclitaxel injection $(10 \mathrm{mg} / \mathrm{kg})$ or paclitaxel nanosuspension $(10 \mathrm{mg} / \mathrm{kg})$ via the tail vein. At predetermined time points $(5,15$, and 30 minutes, and then 1, 2, 4, 8, and 12 hours), four mice from each group were sacrificed, and the liver, kidney, spleen, heart, lung, and brain were collected, washed, weighed, and homogenized in an Ultra Turrax T25 homogenizer (IKA Instruments Ltd) in $1 \mathrm{~mL}$ of salt water. The collected tissue samples were stored at $-80^{\circ} \mathrm{C}$ for further analysis.

To determine the paclitaxel concentration, $50 \mu \mathrm{L}$ anorethisterone $\left(50 \mu \mathrm{g} \cdot \mathrm{mL}^{-1}\right)$ was added to $100 \mu \mathrm{L}$ of tissue homogenate, followed by adding $3 \mathrm{~mL}$ diethyl ether. Each sample was vortexed for 2 minutes. After centrifugation at $4000 \mathrm{rpm}$ for 10 minutes to precipitate the proteins, $1 \mathrm{~mL}$ of the supernatant was removed to clean test tubes and evaporated under a stream of nitrogen at $40^{\circ} \mathrm{C}$. Residues were dissolved in $100 \mu \mathrm{L}$ of mobile phase, and $20 \mu \mathrm{L}$ aliquots were injected into the HPLC for analysis.

\section{HPLC analysis}

The HPLC system composed of a Shimadzu pump (LC20ATvp; Kyoto, Japan), an autosampler, and a wavelength detector was used with data acquisition by LCsolution Lite software (Shimadzu). The HPLC separation was performed on a Dikma Diamonsil $\mathrm{C}_{18}$ analytical column $(150 \mathrm{~mm} \times 4.6 \mathrm{~mm}$, particle size $5 \mu \mathrm{m})$ (Dikma Co, Beijing, China) and a $\mathrm{C}_{18}$ precolumn of the same packing $(10 \mathrm{~mm} \times 4.6 \mathrm{~mm})$. The mobile phase consisted of methanol, acetonitrile, and water (40:34:26, v/v). It was filtered through a $0.45 \mu \mathrm{m}$ nylon filter and degassed in an ultrasonic bath (Kunshan Ultrasonic Instruments Co Ltd, Shanghai, China) before use. All samples were analyzed under isocratic elution at a flow rate of $1 \mathrm{~mL} / \mathrm{min}$ and at a wavelength of $228 \mathrm{~nm}$. The column temperature was maintained at $30^{\circ} \mathrm{C}$.
The HPLC method was validated for the determination of paclitaxel in biological matrix. The limit of quantification (LOQ) (50 ng/mL in plasma or $100 \mathrm{ng} / \mathrm{mL}$ in tissue homogenate), which was determined as the paclitaxel peak, was identifiable and reproducible. A calibration curve was prepared using seven calibration standards $(0.1-40.0 \mu \mathrm{g} / \mathrm{mL}$ in plasma or $0.1-120.0 \mu \mathrm{g} / \mathrm{mL}$ in tissue homogenate). Intraday and interday accuracy and precision were determined by analysis of the $0.5,5.0$, and $20.0 \mu \mathrm{g} / \mathrm{mL}$ concentrations in plasma and $0.5,5.0$, and $60.0 \mu \mathrm{g} / \mathrm{mL}$ concentrations in tissue homogenate. Arterial spin labeling (ASL) relative recoveries from plasma and tissues were determined by comparing the concentration of extracted samples $(0.5,5.0$, and $20.0 \mu \mathrm{g} / \mathrm{mL}$ in plasma and $0.5,5.0$, and $20.0 \mu \mathrm{g} / \mathrm{mL}$ in tissue homogenate) with the unextracted standards containing the same amount of the analyte. In all the cases, three replicate samples were determined.

\section{Pharmacokinetics and statistical analysis}

Pharmacokinetic analysis was carried out using 3P97 pharmacokinetic software (Chinese Pharmacological Association, Beijing, China). Model discrimination was based on Akaike information criteria (AIC). The area under the plasma concentration-time profiles (AUC), the distribution half-life $\left(t_{1 / 2, \alpha}\right)$, the elimination half-life $\left(t_{1 / 2, \beta}\right)$, the mean residence time (MRT), and total plasma clearance (CL) were calculated. The areas under the tissue distribution curves were calculated by the log-linear trapezoidal method. Statistical significance on pharmacokinetic parameter differences among the treatment groups were analyzed by Student's $t$-test using SPSS Statistics (v 11.0; IBM Corporation, Somers, NY), and statistical significance was defined by $P<0.05$.

\section{Results \\ Selection of the surfactants}

Surfactants were the most important factor maintaining the stability of paclitaxel nanosuspensions after the high-pressure homogenization. The surfactants were selected from the general and safe surfactants such as poloxamer 188, PEG-400, CMC-Na, Tween 80, lecithin, tyloxapol, and carbomer.

As seen in Tables 1 and 2, the particle size of paclitaxel nanosuspension changed much according to different surfactants. The nanosuspension prepared with lecithin flocculated quickly, and the results of particle size could not be determined. PEG-400, Tween 80, and tyloxapol were not eligible based on the results of particle size, and ZP values were lower than $|30 \mathrm{mV}|$, indicating electrostatic instability. All paclitaxel nanosuspension prepared with one surfactant flocculated after 4 weeks under room temperature. 
Table I The influence of different surfactants on the particle size, PI, and zeta potential of paclitaxel nanosuspension

\begin{tabular}{lllllll}
\hline Parameter & Carbomer & Poloxamer I88 & PEG-400 & Tween $^{\circledR}$ 80 & CMC-Na & Tyloxapol \\
\hline Particle size, $\mathrm{nm}$ & $255.9 \pm 17.5$ & $294.9 \pm 25.9$ & $278.9 \pm 45.8$ & $334.2 \pm 21.1$ & $735.5 \pm 66.4$ & $339.9 \pm 32.4$ \\
PI & $0.267 \pm 0.04$ & $0.143 \pm 0.08$ & $0.151 \pm 0.08$ & $0.204 \pm 0.07$ & $0.302 \pm 0.11$ & $0.124 \pm 0.07$ \\
Zeta potential, $\mathrm{mV}$ & $-34.9 \pm 5.3$ & $-31.6 \pm 8.6$ & $-27.4 \pm 8.7$ & $-18.5 \pm 8.8$ & $-60 \pm 14.6$ & $-7.4 \pm 2.1$ \\
\hline
\end{tabular}

Abbreviations: CMC-Na, sodium carboxymethyl cellulose; PEG-400, polyethylene glycol 400; PI, polydispersity index.

As seen in Table 3, the combination of two surfactants enhanced the stability of paclitaxel nanosuspension based on the ZP values. The smallest particle size was obtained with the combination of poloxamer 188 and PEG-400, which were selected as the surfactants.

The influence of the proportion of poloxamer 188 and PEG-400 was investigated at the same experiment condition when the concentrations of paclitaxel and surfactant were fixed $(0.04 \%$ and $0.08 \%, w / v)$. As shown in Figure 1, the proportion extent 1:1-1:2 was suitable.

The influence of the proportion of surfactants and paclitaxel on particle size was also investigated when concentration of paclitaxel was fixed $(0.04 \%$, w $/ \mathrm{v})$. As seen in Figure 2, an increased drug concentration needed a corresponding increase of the proportion. The optimized proportion was 1:1-3:1.

\section{Process parameters: homogenization pressure and cycles}

The mean particle size and PI of paclitaxel nanosuspensions were determined by photon correlation spectroscopy to qualify the productions. The influence of homogenization pressure and cycles on the particle size and PI were shown in Figures 3 and 4. The high-pressure homogenization with increased cycle numbers resulted in the formation of smaller paclitaxel particles and PI. The particle size and PI further decreased during 6-20 cycles. However, experiments with homogenization cycles increased 25 from 20 failed to provide further decrease in particle size, which elucidated that the suspensions could not become smaller but could become more uniform during these cycles. To avoid blockage of homogenizer gap and chamber, the paclitaxel coarse powder was homogenized at 300,500 , and 800 bar pressure for six cycles respectively for pre-milling. The homogenization pressure was then increased to 1000 bar for 20 cycles until an equilibrium size was reached.

\section{Paclitaxel nanosuspension}

After the homogenization step, the nanosuspension was immediately lyophilized to obtain the dried paclitaxel nanosuspension which maintained both the physical and chemical stability of the drug. There was no significant change in the paclitaxel nanosuspension size after re-dispersion of the lyophilized particles in glycerin-water. As seen in Figure 5, the form of the paclitaxel nanosuspension was regular rodshaped crystals.

\section{Crystalline state evaluation}

Crystalline state evaluation was carried out after the highpressure homogenization process. As can be seen from the DSC thermograms (Figure 6), the peaks for raw material of paclitaxel and paclitaxel nanoparticles were identical, with two typical melting points, which indicated that the crystalline state was apparently unaltered following the homogenization operation.

\section{Dissolution}

The dissolution profiles for paclitaxel in PBS $(\mathrm{pH}$ 7.4) after high-pressure homogenization are shown in (Figure 7). A 91\% drug dissolution was achieved after the high-pressure homogenization, compared with less than 5\% of raw material.

\section{Stability of nanosuspension}

The physical stability of lyophilized paclitaxel nanosuspension was evaluated over 3 months at room temperature. During this storage period, the particle size did not change,

Table 2 The influence of different surfactants on the stability of paclitaxel nanosuspension

\begin{tabular}{lllllll}
\hline Time & Carbomer & Poloxamer I88 & PEG-400 & Tween $^{\circledR}$ 80 & CMC-Na & Tyloxapol \\
\hline 0 & $255.9 \pm 17.7$ & $294.9 \pm 23.7$ & $278.9 \pm 20.1$ & $334.2 \pm 31.0$ & $735.5 \pm 57.3$ & $339.9 \pm 34.8$ \\
2 weeks & $268.9 \pm 22.6$ & $305.7 \pm 16.2$ & $296.2 \pm 34.7$ & $375.5 \pm 22.9$ & $620.3 \pm 67.9$ & $317.2 \pm 24.5$ \\
3 weeks & $253.2 \pm 14.6$ & $329.9 \pm 27.9$ & $272.5 \pm 12.5$ & $338.7 \pm 38.4$ & $517.7 \pm 47.6$ & $354.7 \pm 33.1$ \\
\hline
\end{tabular}

Abbreviations: CMC-Na, sodium carboxymethyl cellulose; PEG-400, polyethylene glycol 400. 
Table 3 The influence of combinatorial surfactants on the size, PI, and zeta potential of paclitaxel nanosuspension

\begin{tabular}{lclllll}
\hline Parameter & $\begin{array}{l}\text { Carbomer }+ \\
\text { tyloxapol }\end{array}$ & $\begin{array}{l}\text { Carbomer }+ \\
\text { poloxamer } 188\end{array}$ & $\begin{array}{l}\text { Carbomer }+ \\
\text { PEG-400 }\end{array}$ & $\begin{array}{l}\text { Tyloxapol }+ \\
\text { poloxamer I88 }\end{array}$ & $\begin{array}{l}\text { Poloxamer I88+ } \\
\text { PEG-400 }\end{array}$ & $\begin{array}{l}\text { Poloxamer I88 }+ \\
\text { CMC-Na }\end{array}$ \\
\hline Particle size, $\mathrm{nm}$ & $314.1 \pm 13.4$ & $336.4 \pm 13.5$ & $255.7 \pm 14.8$ & $330.6 \pm 21.5$ & $210.6 \pm 16.8$ & $376.2 \pm 49.5$ \\
PI & $0.21 \pm 0.06$ & $0.26 \pm 0.04$ & $0.26 \pm 0.05$ & $0.12 \pm 0.03$ & $0.16 \pm 0.06$ & $0.35 \pm 0.08$ \\
Zeta potential, $\mathrm{mV}$ & $-33.4 \pm 4.8$ & $-36.3 \pm 5.6$ & $-34.8 \pm 6.4$ & $-22.6 \pm 4.7$ & $-30.3 \pm 5.3$ & $-45.1 \pm 11.8$ \\
\hline
\end{tabular}

Abbreviations: CMC-Na, sodium carboxymethyl cellulose; PEG-400, polyethylene glycol 400; PI polydispersity index.

and paclitaxel stability was maintained with more than $99 \%$ of paclitaxel remaining in the nanosuspension. The phenomenon indicated that the lyophilized product had a shelf-life of at least 3 months.

\section{Pharmacokinetics}

The analytical method for paclitaxel determination in plasma has been established. ${ }^{23,24}$ The LOQ for the quantification of paclitaxel was $50 \mathrm{ng} / \mathrm{mL}$ in plasma. The linear range was $0.1-40.0 \mu \mathrm{g} / \mathrm{mL}\left(\mathrm{R}^{2}=0.9992\right)$ in plasma. Intraday and interday precision was $3.3 \%$ and $3.7 \%$, respectively. Paclitaxel relative recoveries from the plasma were $97.7 \%$.

The plasma concentration-time profiles of paclitaxel obtained are shown in Figure 8, and the corresponding pharmacokinetic parameters are shown in Table 4. The pharmacokinetics of paclitaxel was best described by a two-compartment model as shown by the lower AIC and higher $r^{2}$ values. Although both of the plasma profiles declined in a bi-exponential fashion, their profiles were markedly different with paclitaxel nanosuspension exhibiting a very rapid distribution phase $\left(t_{1 / 2, \alpha}=0.186 \pm 0.077\right.$ hours $)$ compared with paclitaxel injection $\left(t_{1 / 2, \alpha}=0.495 \pm 0.114\right.$ hours $)$. In contrast, the elimination phase $\left(t_{1 / 2, \beta}\right)$ for the paclitaxel nanosuspension formulation was longer $\left(t_{1 / 2, \beta}=5.645 \pm 2.941\right.$ hours $)$ compared with paclitaxel injection $\left(t_{1 / 2, \beta}=3.774 \pm 1.352\right.$ hours $)$. The plasma $\mathrm{AUC}_{0-\infty}$

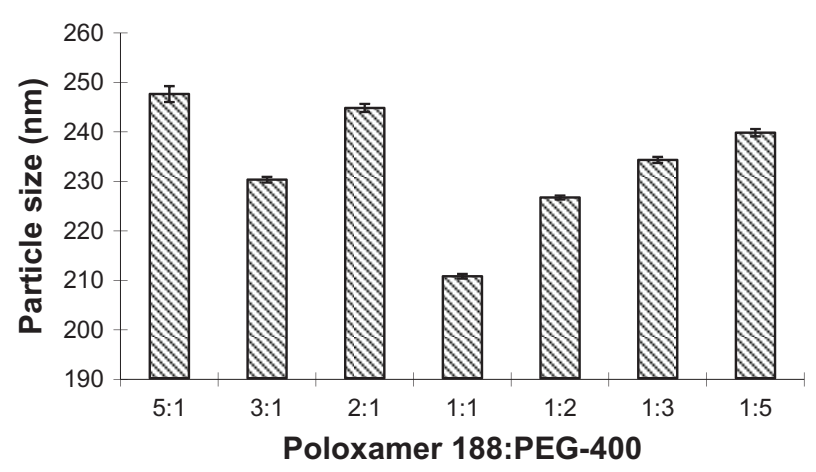

Figure I The influence of different ratios of poloxamer 188 and PEG-400 on the particle size.

Abbreviation: PEG-400, polyethylene glycol 400. for paclitaxel injection solution $\left(20.343 \pm 9.119 \mu \mathrm{g} \cdot \mathrm{h} \cdot \mathrm{mL}^{-1}\right)$ was approximately four-fold greater than paclitaxel nanosuspension $\left(5.196 \pm 1.426 \mu \mathrm{g} \cdot \mathrm{h} \cdot \mathrm{mL}^{-1}\right)$, but the MRT ( $3.199 \pm 3.718$ hours) for the paclitaxel nanosuspension formulation was longer than that observed for the paclitaxel injection solution ( $2.753 \pm 0.943$ hours). In rat plasma, paclitaxel was measurable at 24 hours after injection of paclitaxel nanosuspension, but only up to 12 hours after intravenous paclitaxel injection solution. Thus, it was apparent that the paclitaxel nanosuspension formulation maintained lower plasma paclitaxel concentrations but for a longer duration compared with the paclitaxel injection solution.

\section{Tissue distribution}

The analytical method for paclitaxel determination in tissue homogenate has been established. ${ }^{24}$ The LOQ for the quantification of paclitaxel was $100 \mathrm{ng} / \mathrm{mL}$ in tissue homogenate (heart, liver, spleen, lung, kidney, brain). The linear range was $0.1-120.0 \mu \mathrm{g} / \mathrm{g}\left(\mathrm{R}^{2}\right.$ was $\left.0.9921-0.9996\right)$, respectively. Intraday and interday precision was $3.1 \%-4.7 \%$ and $4.0 \%-5.11 \%$, respectively. Paclitaxel relative recoveries from the plasma were $96.9 \%-98.4 \%$, respectively.

In contrast to the plasma profiles, in all tissues except the heart and kidney, significantly higher paclitaxel concentrations were paclitaxel injection solution $(P<0.001)$ (Figure 9). The increase in $\mathrm{AUC}_{0-\infty}$ ranged from 2.38-fold

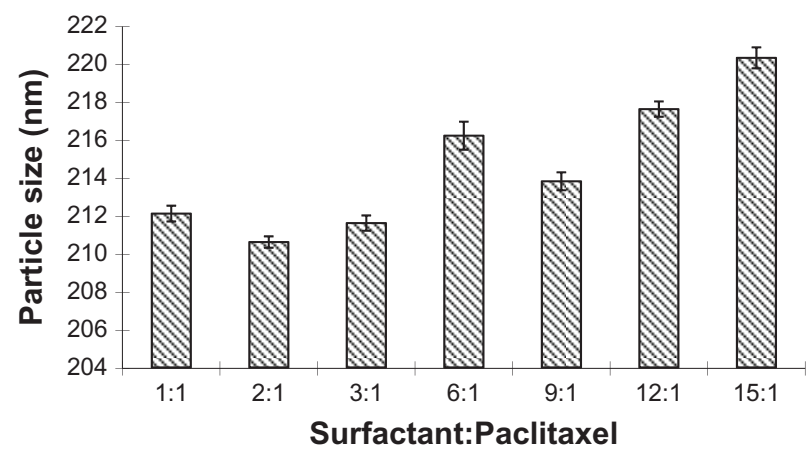

Figure 2 The influence of different ratios of surfactant and paclitaxel on the size of nanoparticles. 


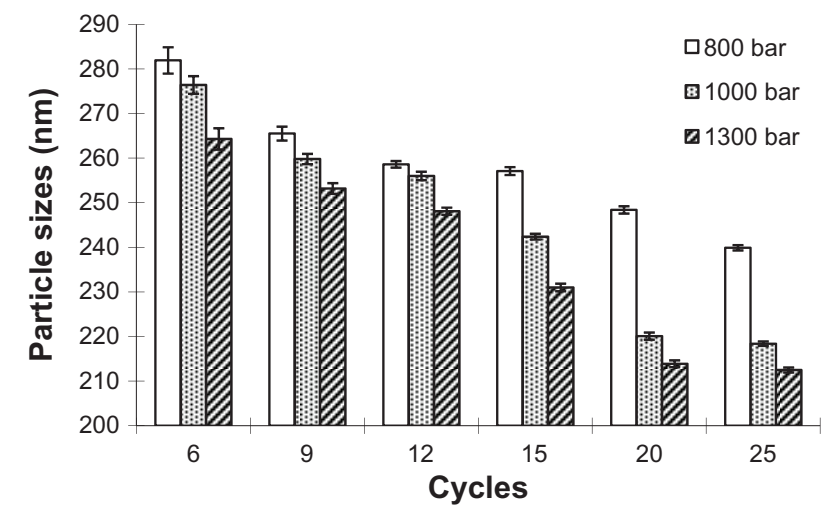

Figure 3 The influence of homogenization pressure and cycle number on the particle size of nanosuspension.

in the lung to 11.45 -fold in the spleen, whereas the heart experienced a $19 \%$ reduction (Table 5 ).

\section{Discussion}

DSC (Figure 6) profiles of paclitaxel nanoparticles were not affected, indicating that the crystalline state of paclitaxel appeared to be unaltered following the homogenization operation, which is important for long-term stability. The two melting peaks of paclitaxel nanoparticles were slightly ahead of the peaks of raw material, possibly because the nanosuspension had smaller particle size and larger surface area. When the outside temperature rose, the rate of heat transfer from surface to the center of paclitaxel nanoparticles was higher than that of paclitaxel raw material, which made the nanosuspension more sensitive to external temperature.

The plasma pharmacokinetics of paclitaxel given in the nanosuspension formulation was markedly different compared with the paclitaxel injection solution. The in vivo properties

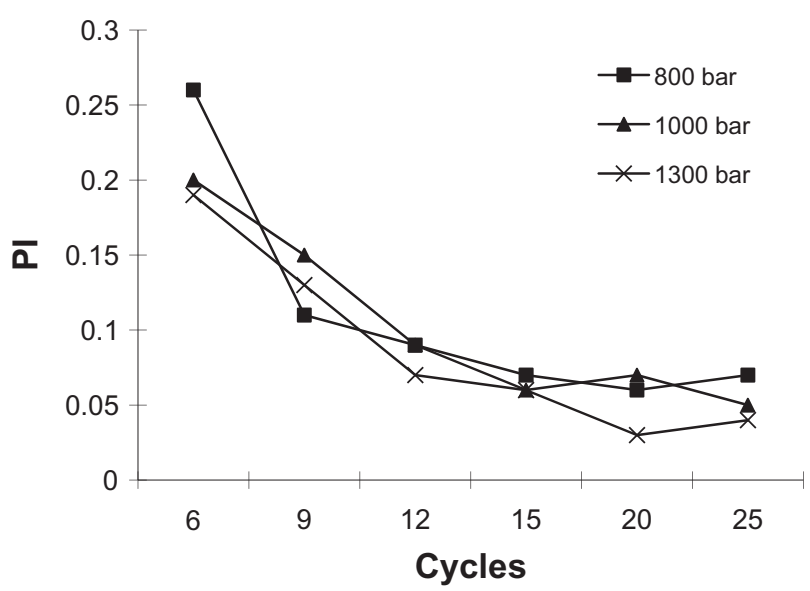

Figure 4 The influence of homogenization pressure and cycle number on the $\mathrm{Pl}$ of nanoparticles (standard deviation values are not reported). Abbreviation: PI, polydispersity index. of a nanosuspension formulation strongly depend on the nanoparticle size, dissolution rate, and nature. ${ }^{13}$ Particles that dissolve rapidly in the blood would be expected to exhibit similar pharmacokinetic behavior to a drug in solution form. This was observed with flurbiprofen nanosuspension, which has similar pharmacokinetics and tissue distribution as a flurbiprofen solution after intravenous administration in rats. ${ }^{25} \mathrm{It}$ is thought to be due to the rapid dissolution of the flurbiprofen nanoparticles in the bloodstream. The in vitro dissolution results (Figure 7) indicated that the paclitaxel nanoparticles might dissolve in the blood quickly, which was consistent with the above theory. Although the plasma AUC of paclitaxel nanosuspension was less than $30 \%$ of the AUC of the paclitaxel injection solution, the plasma profiles indicated that the nanosuspension formulation maintained low plasma drug for a longer period of time. The results were consistent with the study of asulacrine nanosuspension. ${ }^{26}$

The pharmacokinetic profile in rats after intravenous administration of paclitaxel nanosuspension dropped rapidly in plasma concentration to $2 \mu \mathrm{g} / \mathrm{mL}$ in 30 minutes. We suggested that this might be due to the rapid uptake of rather slowly dissolving paclitaxel nanoparticles by the reticuloendothelial system (RES). Previous studies have shown that slow dissolving nanocrystals were taken up by the phagocytic cells of the mononuclear phagocyte system (MPS), primarily the Kupper cells in the liver, spleen, and lung. ${ }^{26,27}$ Their lipophilic character might permit passage through the

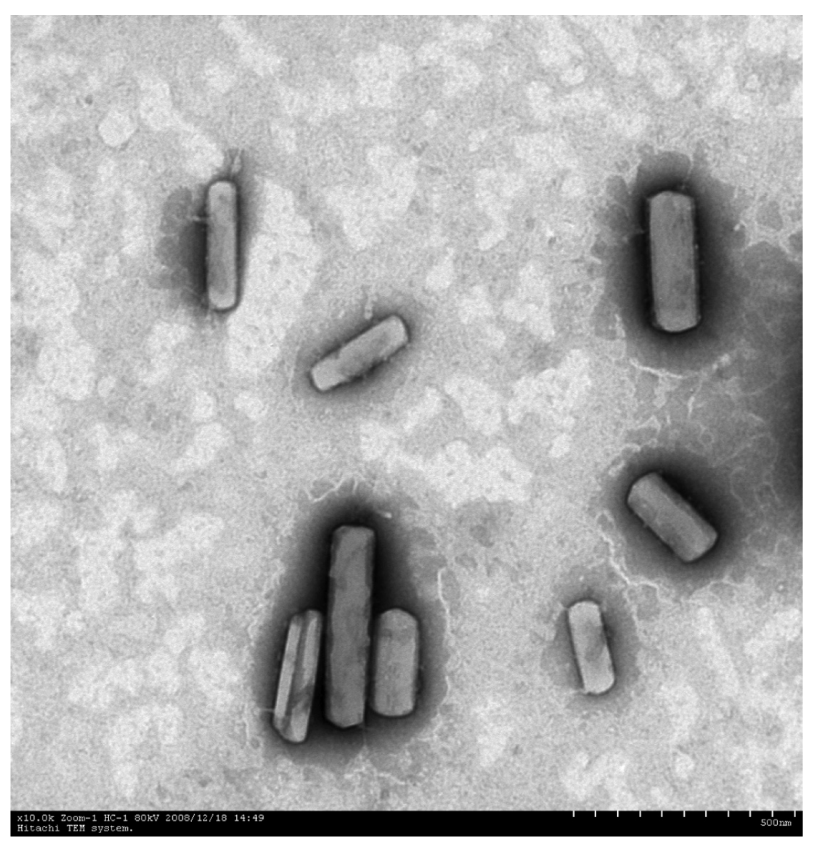

Figure 5 Transmission electron microscopy of paclitaxel nanosuspension. Magnification: 10,000x. 

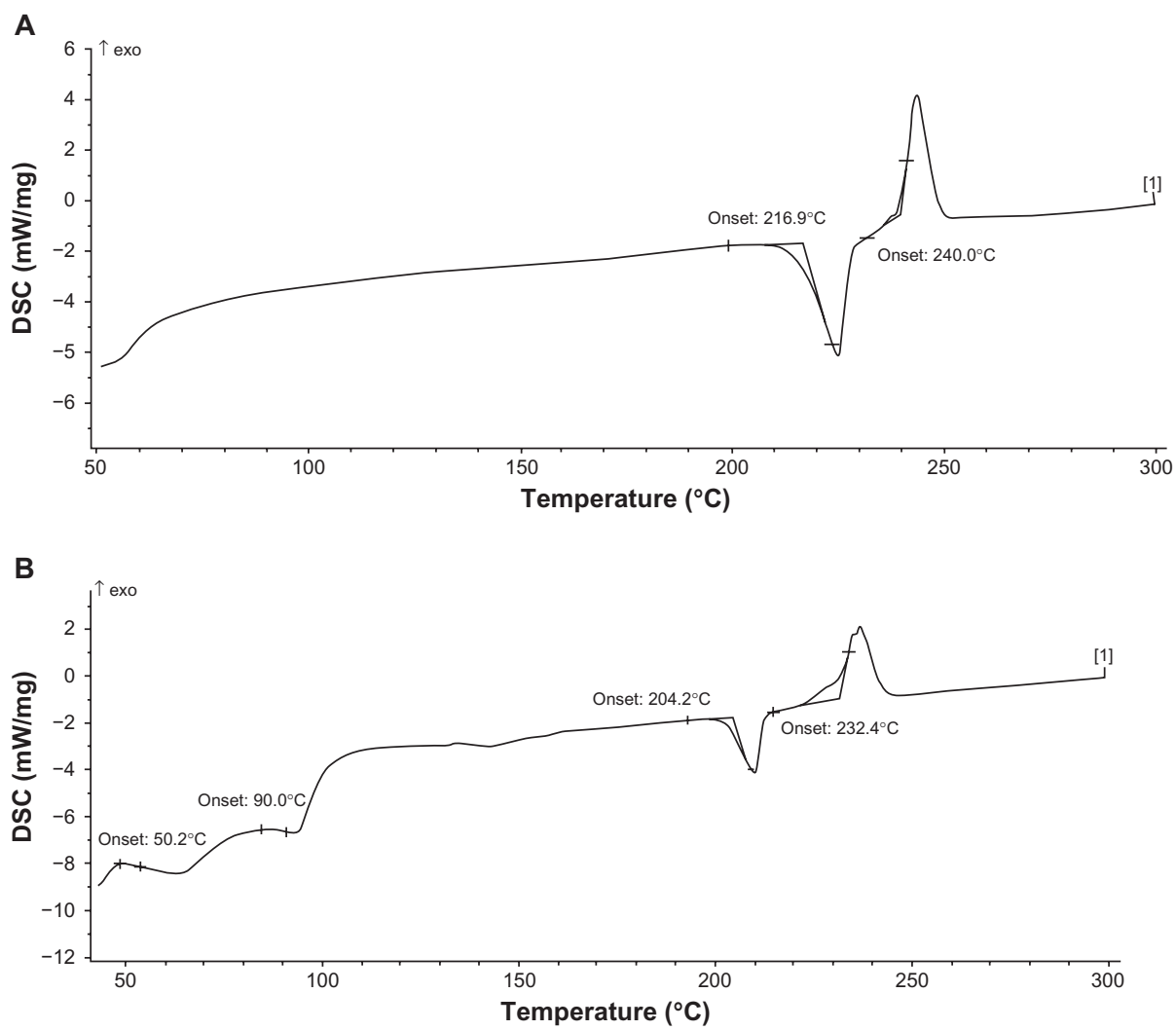

Figure 6 Differential scanning capacity thermography. (A) Raw material of paclitaxel. (B) Paclitaxel nanosuspension.

phagolysosomal membrane, and as a result they can leave the cellular vesicle, enter the cytoplasm, and then excite the cell by diffusing down the drug concentration gradient. ${ }^{13}$ This effect would result in a pharmacokinetic profile with significantly reduced AUC, but prolonged half-life. ${ }^{26}$

The paclitaxel nanosuspension in the phagocytic cells might slowly dissolve and diffuse into the blood circulation to maintain blood concentrations for a longer duration.

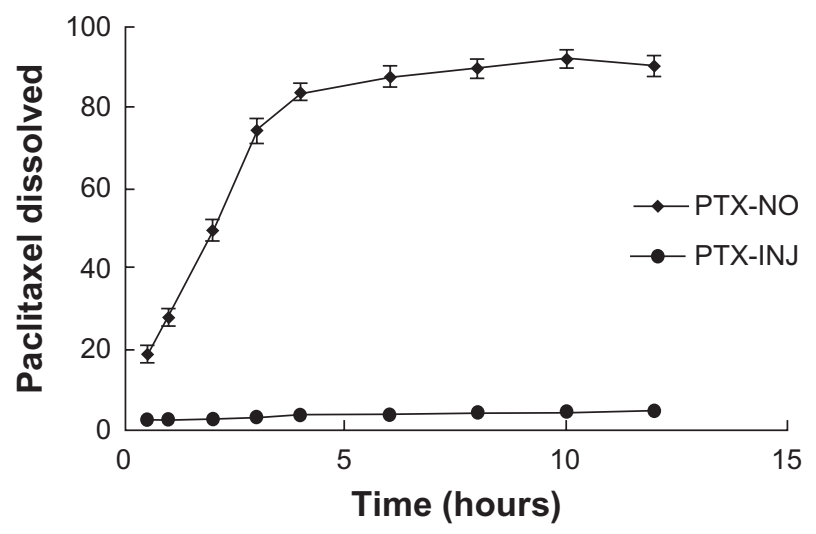

Figure 7 Dissolution profiles of paclitaxel from PTX-NO and PTX-INJ into mice at a dose of $10 \mathrm{mg}$ paclitaxel $/ \mathrm{kg}$.

Note: Data are mean \pm standard deviation, $n=3$.

Abbreviations: PTX-INJ, paclitaxel injection; PTX-NO, paclitaxel nanosuspension.
This could be the reason why a low paclitaxel nanosuspension concentration was observed for a prolonged time after it was given to the rat. Higher plasma clearance and remarkably increased tissue concentrations could also support rapid clearance of paclitaxel from the blood circulation by

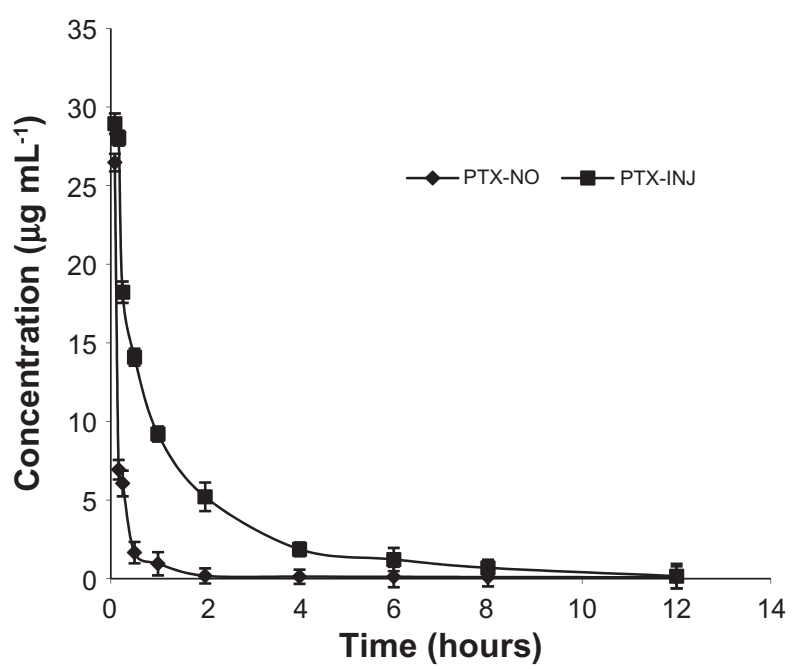

Figure 8 The curves of the mean plasma drug concentration versus time in rats after intravenous injection of PTX-NO and PTX-INJ.

Note: Data are mean \pm standard deviation, $n=3$.

Abbreviations: PTX-INJ, paclitaxel injection; PTX-NO, paclitaxel nanosuspension. 
Table 4 Pharmacokinetic parameters in rats after intravenous injection of PTX-NO and PTX-INJ $(n=6)$

\begin{tabular}{lcr}
\hline Parameters & PTX-NO & PTX-IN J \\
\hline$\alpha / h^{-1}$ & $4.177 \pm 1.537^{\mathrm{a}}$ & $1.466 \pm 0.366$ \\
$\beta / \mathrm{h}^{-1}$ & $0.142 \pm 0.049$ & $0.204 \pm 0.082$ \\
$V_{\mathrm{C}}, \mathrm{L} \cdot \mathrm{kg}^{-1}$ & $0.748 \pm 0.263$ & $0.697 \pm 0.27 \mathrm{I}$ \\
$t_{1 / 2, \alpha}$, hours & $0.1863 \pm 0.077^{\mathrm{a}}$ & $0.495 \pm 0.114$ \\
$t_{1 / 2, \beta}$, hours & $5.646 \pm 2.94 \mathrm{I}$ & $3.774 \pm 1.352$ \\
$\mathrm{AUC}$ & \\
$\mathrm{CL}(\mathrm{s}), \mathrm{L} \cdot \mathrm{Lg} \cdot \mathrm{kg} \cdot \mathrm{mL}^{-1} \cdot \mathrm{h}^{-1}$ & $5.196 \pm 1.426^{\mathrm{b}}$ & $20.343 \pm 9.119$ \\
$\mathrm{MRT}, \mathrm{hours}$ & $2.050 \pm 0.616^{\mathrm{a}}$ & $0.556 \pm 0.190$ \\
\hline
\end{tabular}

Notes: ${ }^{a} P<0.05$ vs PTX-IN]; ${ }^{\text {} P}<0.01$ vs PTX-INJ.

Abbreviations: $\mathrm{AUC}$, area under concentration curve; $\mathrm{CL}$, total plasma clearance; MRT, mean residence time; PTX-INJ, paclitaxel injection; PTX-NO, paclitaxel nanosuspension; $t_{1 / 2, \alpha^{\prime}}$, distribution half-life; $t_{1 / 2,3}$, elimination half-life.

the RES. Despite the enhanced plasma clearance, a longer $t_{1 / 2, \beta}$ for paclitaxel nanosuspension was observed, likely due to the enhanced distribution (11-fold) of the paclitaxel nanosuspension out of the bloodstream and uptake into the tissues such as the liver, kidney, and lung. PEG-modification of the surface of slowly dissolving drug crystals could reduce the macrophage uptake as observed with nanoparticles..$^{28}$ This would increase circulation time and allow the particles to leak out of discontinuities in tumor vasculature. The enhanced accumulation via the enhanced permeability and retention effect resulted in passive targeting. ${ }^{29}$ Therefore, the PEG-modification of paclitaxel nanosuspension would avoid macrophage uptake and allow for longer duration in the blood circulation. As a consequence, enhanced tumor accumulation of the nanocrystals may occur. ${ }^{26}$
For paclitaxel nanosuspension, the drug nanocrystals could circulate in the blood as submicron particles for a certain time period. The nanocrystals might then be recognized as foreign matter and be rapidly cleared by phagocytic cells of MPS which are abundant in special tissues and organs such as liver, spleen, and lung. ${ }^{30}$ It has been shown that the uptake of nanoparticles by RES organs following intravenous administration might take anywhere from a few minutes to hours, depending on particle size and composition. ${ }^{31}$ Therefore, the paclitaxel nanocrystals in paclitaxel nanosuspension had a markedly higher concentration compared with paclitaxel injection solution in these organs. Meanwhile the drug concentration in kidney and heart decreased. Similar results were reported in the literature. ${ }^{18,26} \mathrm{In}$ brain homogenate, paclitaxel was measurable until 12 hours postinjection of paclitaxel nanosuspension, while the concentration was negligible after intravenous paclitaxel injection solution. This phenomenon indicated nanosuspension could easily enter the brain through the blood-brain barrier, which is important for the research of brain targeted therapy.

It is contradictory to uptake the nanocarriers by the RES system while keeping the target character of nanocarriers. The uptake of nanocarriers by RES led to the high drug concentration in liver which indicated that the liver toxicity was theoretically increased. In the pre-experiments of the pharmacokinetic study, all female rats died after the injection of paclitaxel injection $(15 \mathrm{mg} / \mathrm{kg})$, and the male rats showed severe symptoms such as tremors and sticky plasma; while rats injected with paclitaxel nanosuspension $(15 \mathrm{mg} / \mathrm{kg})$ all survived, and there was no significant toxicity reaction. This phenomenon could prove that the nanosuspension has lower
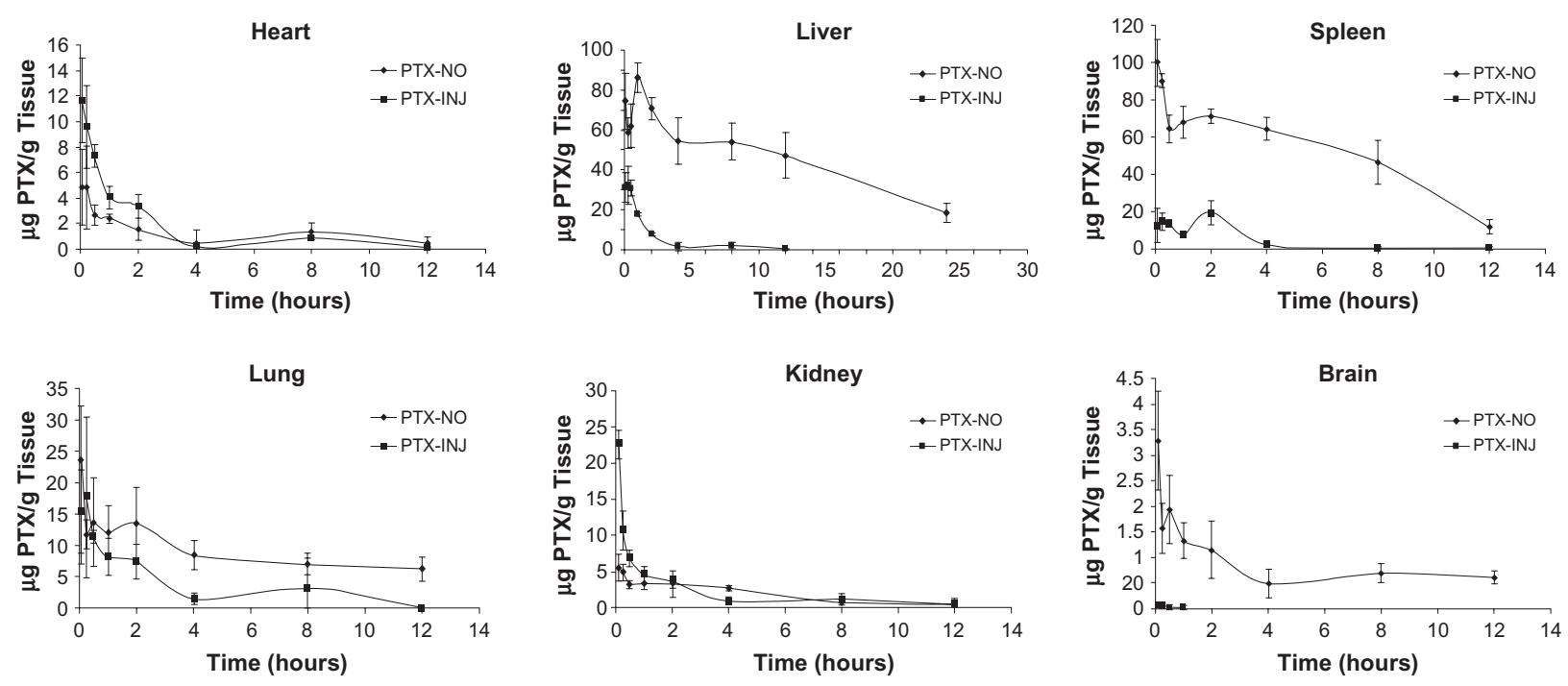

Figure 9 Tissue distribution curves of PTX-NO and PTX-INJ after $10 \mathrm{mg}$ paclitaxel/kg intravenous injection in mice.

Note: Data are mean \pm standard deviation, $n=3$.

Abbreviations: PTX-INJ, paclitaxel injection; PTX-NO, paclitaxel nanosuspension. 
Table 5 AUC values in various tissues after intravenous injection of PTX-NO and PTX-INJ into the mice

\begin{tabular}{lccl}
\hline Tissue & AUC $_{0-12 \mathbf{h}}\left(\mu \mathbf{g} \cdot \mathbf{g}^{-1} \cdot \mathbf{h}\right)$ & & $\mathbf{r}_{\mathrm{e}}^{\mathrm{a}}$ \\
\cline { 2 - 3 } & $\mathbf{P T X - N O}$ & PTX-INJ & \\
\hline Heart & $14.5 \mathrm{I} \pm 6.21$ & $18.02 \pm 5.84$ & $0.8 \mathrm{I}$ \\
Liver & $687.58 \pm 117.56^{\mathrm{b}}$ & $60.45 \pm 20.49$ & 11.37 \\
Spleen & $613.06 \pm 218.34^{\mathrm{b}}$ & $53.52 \pm 29.55$ & 11.45 \\
Lung & $104.48 \pm 24.73^{\mathrm{b}}$ & $43.92 \pm 34.92$ & 2.38 \\
Kidney & $21.91 \pm 10.93$ & $22.68 \pm 9.47$ & 0.97 \\
Brain & $9.48 \pm 3.45$ & $0.03 \pm 0.03$ & 294.27
\end{tabular}

Notes: ${ }^{a} r_{e}=A U C_{\text {PTX-NO }} / A \cup C_{\text {PTX-IN]; }}{ }^{\text {bP }}<0.01$ vs PTX-INJ.

Abbreviations: AUC, area under concentration curve; PTX-IN], paclitaxel injection; PTX-NO, paclitaxel nanosuspension.

toxicity than the paclitaxel injection. The actual toxicity in liver should be proved by long-term toxicity tests. If liver is the target organ of a drug, the drug has potential application in the clinic. In this study, the nanosuspension and injection were administered with equal doses in order to study the different pharmacokinetic characters of paclitaxel. In the pharmacodynamics study, the paclitaxel nanosuspension could be administered with much lower doses to achieve good therapeutic effects for liver cancer, which is one of the main targets of paclitaxel. In low doses, the side effects of paclitaxel should be ignored as the liver concentration of paclitaxel was much higher compared with other organs.

\section{Conclusion}

The nanosuspension formulations of paclitaxel with the smaller particle size could be effectively produced using the high-pressure homogenization method. The method was shown to be simple and adequate for drug particle size reduction and did not seem to alter the crystalline state of the drug, which is important for long-term stability. To overcome particle growth during long-term storage of nanosuspension formulations, lyophilization was carried out in order to assess the feasibility of nanosuspension in dry products. The paclitaxel nanosuspension exhibited a significantly reduced $\mathrm{AUC}_{0-\infty}$, greater CL, shorter $t_{1 / 2, \beta}$ in plasma and resulted in a significantly greater $\mathrm{AUC}_{0-\infty}$ in the liver, lung, and spleen compared with the paclitaxel solution.

\section{Acknowledgments}

The authors gratefully acknowledge the Natural Science Foundation of the Higher Education Institutions of Jiangsu Province, China (10KJB350002) and the State Key Laboratory of Materials-Oriented Chemical Engineering (KL10-13) for the financial support of this work.

\section{Disclosure}

The authors report no competing financial interests in this work.

\section{References}

1. Singla AK, Garg A, Aggarwal D. Paclitaxel and its formulations. Int $J$ Pharm. 2002;235(1-2):179-192.

2. Appendino G. Taxol (paclitaxel): historical and ecological aspects. Fitoterapia. 1993;64(Suppl 1):5-25.

3. Foa R, Norton L, Seidman A. Taxol (paclitaxel): a novel anti-microtubule agent with remarkable anti-neoplastic activity. Int $J$ Clin Lab Res. 1994; 24(1):6-14.

4. Spencer C, Faulds D. Paclitaxel. A review of its pharmacodynamic and pharmacokinetic properties and therapeutic potential in the treatment of cancer. Drugs. 1994;48(5):794.

5. Chervinsky D, Brecher M, Hoelcle M. Cremophor-EL enhances taxol efficacy in a multi-drug resistant $\mathrm{C} 1300$ neuroblastoma cell line. Anticancer Res. 1993;13(1):93-96.

6. Webster L, Linsenmeyer M, Millward M, Morton C, Bishop J, Woodcock D. Measurement of cremophor EL following taxol: plasma levels sufficient to reverse drug exclusion mediated by the multidrugresistant phenotype. J Natl Cancer Inst. 1993;85(20):1685.

7. Fjällskog ML, Frii L, Bergh J. Is Cremophor EL, solvent for paclitaxel, cytotoxic? Lancet. 1993;342(8875):873.

8. Dorr R. Pharmacology and toxicology of Cremophor EL diluent. Ann Pharmacother. 1994;28(5):S11-S14.

9. Rowinsky E, Eisenhauer E, Chaudhry V, Arbuck S, Donehower R. Clinical toxicities encountered with paclitaxel (Taxol). Semin Oncol. 1993;20(4 Suppl 3):1-15.

10. Weiss R, Donehower R, Wiernik P, et al. Hypersensitivity reactions from taxol. J Clin Oncol. 1990;8(7):1263.

11. Böhm BHL, Müller RH. Lab-scale production unit design for nanosuspensions of sparingly soluble cytotoxic drugs. Pharm Sci Technol Today. 1999;2(8):336-339.

12. Patravale V, Date A, Kulkarni R. Nanosuspensions: a promising drug delivery strategy. J Pharm Pharmacol. 2004;56(7):827-840.

13. Rabinow B. Nanosuspensions in drug delivery. Nat Rev Drug Discov. 2004;3(9):785-796.

14. Hintz RJ, Johnson KC. The effect of particle size distribution on dissolution rate and oral absorption. Int J Pharm. 1989;51(1):9-17.

15. Müller RH, Jacobs C, Kayser O. Nanosuspensions as particulate drug formulations in therapy: rationale for development and what we can expect for the future. Adv Drug Deliv Rev. 2001;47(1):3-19.

16. Muller RH, Keck CM. Challenges and solutions for the delivery of biotech drugs - a review of drug nanocrystal technology and lipid nanoparticles. J Biotechnol. 2004;113(1-3):151-170.

17. Müller RH, Peters K. Nanosuspensions for the formulation of poorly soluble drugs: I. Preparation by a size-reduction technique. Int J Pharm. 1998;160(2):229-237.

18. Peters K, Leitzke S, Diederichs J, et al. Preparation of a clofazimine nanosuspension for intravenous use and evaluation of its therapeutic efficacy in murine Mycobacterium avium infection. $J$ Antimicrob Chemother. 2000;45(1):77.

19. Merisko-Liversidge E, Sarpotdar P, Bruno J, et al. Formulation and antitumor activity evaluation of nanocrystalline suspensions of poorly soluble anticancer drugs. Pharm Res. 1996;13(2):272-278.

20. Li X, Gu L, Xu Y, Wang Y. Preparation of fenofibrate nanosuspension and study of its pharmacokinetic behavior in rats. Drug Dev Ind Pharm. 2009;35(7):827-833.

21. Deng J, Huang L, Liu F. Understanding the structure and stability of paclitaxel nanocrystals. Int J Pharm. 2010;390(2):242-249.

22. Mishra PR, Shaal LA, Müller RH, Keck CM. Production and characterization of Hesperetin nanosuspensions for dermal delivery. Int $J$ Pharm. 2009;371(1-2):182-189. 
23. Rouini M, Lotfolahi A, Stewart D, et al. A rapid reversed phase high performance liquid chromatographic method for the determination of docetaxel (Taxotere ${ }^{\mathbb{R}}$ ) in human plasma using a column switching technique. J Pharm Biomed Anal. 1998;17(8):1243-1247.

24. Sparreboom A, van Tellingen O, Noojien WJ, Beijnen JH. Determination of paclitaxel and metabolites in mouse plasma, tissues, urine and faeces by semi-automated reversed-phase high-performance liquid chromatography. J Chromatogr B Biomed Sci Appl. 1995;664(2):383-391.

25. Clement M, Pugh W, Parikh I. Tissue distribution and plasma clearance of a novel microcrystalline-coated flurbiprofen formulation. Pharmacologist. 1992;34:204.

26. Ganta S, Paxton JW, Baguley BC, Garg S. Formulation and pharmacokinetic evaluation of an asulacrine nanocrystalline suspension for intravenous delivery. Int J Pharm. 2009;367(1-2):179-186.

27. Moghimi S, Hunter A, Murray J. Long-circulating and target-specific nanoparticles: theory to practice. Pharmacol Rev. 2001;53(2):283.
28. Devalapally H, Shenoy D, Little S, Langer R, Amiji M. Poly (ethylene oxide)-modified poly (beta-amino ester) nanoparticles as a $\mathrm{pH}$-sensitive system for tumor-targeted delivery of hydrophobic drugs: part 3. Therapeutic efficacy and safety studies in ovarian cancer xenograft model. Cancer Chemother Pharmacol. 2007;59(4): 477-484.

29. Maeda H, Matsumura Y. Tumoritropic and lymphotropic principles of macromolecular drugs. Critical reviews in therapeutic drug carrier systems. 1989;6(3):193.

30. Gao L, Zhang D, Chen M, et al. Studies on pharmacokinetics and tissue distribution of oridonin nanosuspensions. Int J Pharm. 2008;355(1-2):321-327.

31. Manjunath K, Venkateswarlu V. Pharmacokinetics, tissue distribution and bioavailability of clozapine solid lipid nanoparticles after intravenous and intraduodenal administration. $J$ Control Release. 2005;107(2):215-228.
International Journal of Nanomedicine

\section{Publish your work in this journal}

The International Journal of Nanomedicine is an international, peerreviewed journal focusing on the application of nanotechnology in diagnostics, therapeutics, and drug delivery systems throughout the biomedical field. This journal is indexed on PubMed Central,

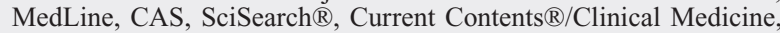

\section{Dovepress}

Journal Citation Reports/Science Edition, EMBase, Scopus and the Elsevier Bibliographic databases. The manuscript management system is completely online and includes a very quick and fair peer-review system, which is all easy to use. Visit http://www.dovepress.com/ testimonials.php to read real quotes from published authors.

Submit your manuscript here: http://www.dovepress.com/international-journal-of-nanomedicine-journal 